Review

\title{
Catalyst-Controlled Site-Selectivity Switching in Pd-Catalyzed Cross-Coupling of Dihaloarenes
}

\section{Kei Manabe * and Miyuki Yamaguchi}

School of Pharmaceutical Sciences, University of Shizuoka, 52-1 Yada, Suruga-ku, Shizuoka 422-8526, Japan; E-Mail: yamaguchim@u-shizuoka-ken.ac.jp

* Author to whom correspondence should be addressed; E-Mail: manabe@u-shizuoka-ken.ac.jp; Tel.: +81-54-264-5754; Fax: +81-54-264-5586.

Received: 29 May 2014; in revised form: 28 July 2014 / Accepted: 5 August 2014 /

Published: 20 August 2014

\begin{abstract}
Pd-catalyzed, site-selective mono-cross-coupling of substrates with two identical halo groups is a useful method for synthesizing substituted monohalogenated arenes. Such arenes constitute an important class of compounds, which are commonly identified as drug components and synthetic intermediates. Traditionally, these site-selective reactions have been realized in a "substrate-controlled" manner, which is based on the steric and electronic differences between the two carbon-halogen bonds of the substrate. Recently, an alternative strategy, "catalyst-controlled" site-selective cross-coupling, has emerged. In this strategy, the preferred reaction site of a dihaloarene can be switched, merely by changing the catalyst used. This type of selective reaction further expands the utility of Pd-catalyzed cross-coupling. In this review, we summarize the reported examples of catalyst-controlled site-selectivity switching in Pd-catalyzed cross-coupling of dihaloarenes.
\end{abstract}

Keywords: palladium; Suzuki-Miyaura coupling; site-selectivity; Grignard reagent; phosphine

\section{Introduction}

Pd-catalyzed cross-coupling of haloarenes (or pseudo-haloarenes, such as aryl triflates) with organometallic reagents constitutes one of the most important and practical reactions in transition metal-catalyzed carbon-carbon bond formations [1-3]. The cross-coupling is so reliable, that it has been applied to a wide variety of substrates. Among them, dihaloarenes have attracted considerable interest as important substrates, because mono-cross-coupling of dihaloarenes affords monohaloarenes. 
Monohaloarenes are not only versatile synthetic intermediates for the synthesis of multisubstituted arenes, but they are also themselves important structural motifs in valuable compounds, including many pharmaceuticals. An important issue to be addressed in the mono-cross-coupling of dihaloarenes is the selectivity between the two halo groups. In general, the intrinsic difference in the reactivity of the halo groups (i.e., $\mathrm{I}>\mathrm{Br}>\mathrm{Cl}>\mathrm{F}$ ) can be utilized to realize chemoselective cross-coupling at the desired position [4-7]. Interesting examples of chemoselectivity controlled by the catalysts used have been also reported [8-10]. There are also examples of chemoselectivity between two nucleophilic groups [11]. In comparison with the chemoselective cross-coupling, it is difficult to achieve siteselective cross-coupling of substrates where both halo groups are the same. Nevertheless, because these substrates can generally be prepared in shorter steps than the compounds with two different halo groups, this type of site-selective cross-coupling can prove more useful for the synthesis of multisubstituted arenes [12-15]. Therefore, it is highly desirable to develop efficient site-selective cross-coupling of dihaloarenes.

The majority of examples for the site-selective cross-coupling of dihaloarenes reported so far are based on the "substrate-controlled" strategy, which relies on the different reactivity of the two carbon-halogen bonds in the substrate. Generally, as expected, the reaction selectively occurs at the less sterically hindered halo group. These reactions are also governed by electronic effects. For dihalobenzenes, the reaction preferentially occurs at the carbon-halogen bond bearing the less electron-rich carbon [16]. For dihaloheteroarenes, detailed studies have been conducted [17], and the site-selectivity has been found to be determined by factors, such as the strengths of the carbon-halogen bonds and the coefficients of the heterocycle $\pi^{*}$ (LUMO) $[18,19]$.

Recently, an alternative "catalyst-controlled" strategy for site-selective cross-coupling has emerged. In this strategy, the reaction site of a dihaloarene can be controlled by the catalyst used, regardless of the intrinsic reactivity of the halo groups in the substrate. Furthermore, it is possible to switch the site-selectivity merely by changing the catalyst used; different products can be obtained selectively starting from a common dihaloarene (Scheme 1). This strategy allows for the rapid preparation of diverse multisubstituted arenes and greatly expands the synthetic utility of dihaloarenes.

Scheme 1. Catalyst-controlled site-selectivity switching in cross-coupling of dihaloarenes. $X=$ halogen; $M=\mathrm{MgBr}, \mathrm{B}(\mathrm{OH})_{2}$, etc.

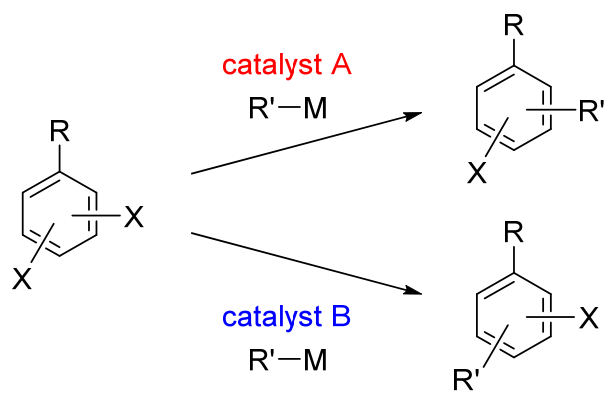

Pd catalysts have been used for the catalyst-controlled switching of site-selectivity. In many cases, the choice of appropriate catalyst ligands is the key to controlling site-selectivity. These ligands coordinate with palladium and affect which of the two carbon-halogen bonds is preferred in oxidative addition, the step generally considered to be the irreversible, selectivity-determining step in these cross-coupling 
reactions (Scheme 2), although reversible oxidative addition was also observed in some cases [20]. While the reasons for this selectivity during oxidative addition are, in most cases, unclear at this moment, we believe that summarizing the examples will help researchers to appreciate the wide applicability of this approach and develop new examples of this useful strategy. Thus, in this review, we summarize the examples of the catalyst-controlled site-selective cross-coupling in which selectivity switching was observed by changing the catalyst. The examples are categorized based on the substrates used.

Scheme 2. General catalytic cycle of the Pd-catalyzed cross-coupling of dihaloarenes. $\mathrm{L}=$ ligand.

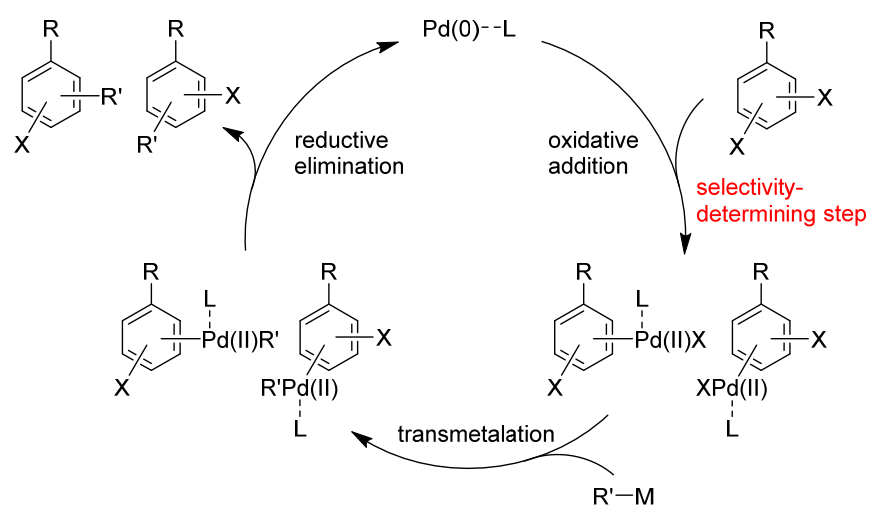

\section{Dihalobenzenes}

\subsection{Phenol Derivatives}

In 2007, we reported the first example of catalyst-controlled site-selective cross-coupling of dihalophenols [21]. When Kumada-Tamao-Corriu coupling [22,23] of 2,4-dibromophenol (1) with an excess of Grignard reagent 2 was conducted in the presence of tris(dibenzylideneacetone)dipalladium $\left(\mathrm{Pd}_{2}(\mathrm{dba})_{3}\right)$ and a hydroxyterphenylphosphine, Ph-HTP, which we developed [24,25], product 3 was selectively obtained in a good yield (Scheme 3). On the other hand, when the Ph-HTP ligand was replaced with 1,1'-bis(diphenylphosphino)ferrocene (DPPF), isomer 4 was selectively produced. Thus, the site-selectivity was well controlled by the ligand used. Furthermore, di-cross-coupling, in which both the bromo groups were substituted with 4-methoxyphenyl groups, occurred to a very small extent (giving the corresponding product in $<5 \%$ yield) in spite of the presence of excess 2.

Scheme 3. Site-selective cross-coupling of 2,4-dibromophenol with a Grignard reagent. DPPF, 1,1'-bis(diphenylphosphino)ferrocene; Ph-HTP, hydroxyterphenylphosphines.

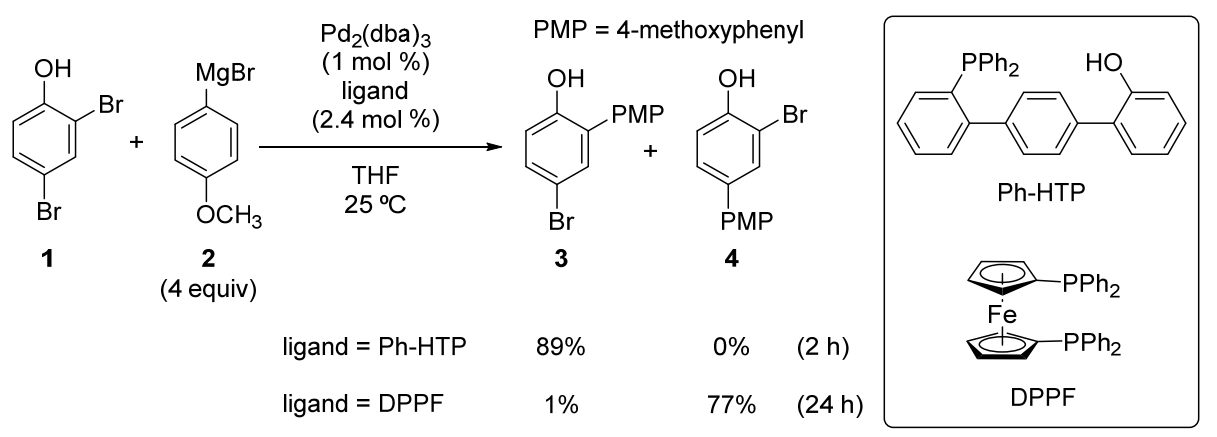


The selective formation of $\mathbf{4}$ induced by the DPPF-based catalyst can be attributed to less steric hindrance for the oxidative addition at the position para to the hydroxy group. On the other hand, the highly selective formation of $\mathbf{3}$ induced by the Ph-HTP-based catalyst cannot be explained by steric effects. The hydroxy group of the substrate and the ligand were essential for the high yield and selectivity; both of the corresponding methoxy derivatives of Ph-HTP and the substrate did not show high selectivity. We assume the following mechanism for the site-selective cross-coupling at the ortho position (Scheme 4). Ph-HTP is deprotonated by Grignard reagent and, in the presence of palladium, presumably forms palladium/magnesium bimetallic species A. This species and the substrate, which also exists as a magnesium salt, are in equilibrium with magnesium bisphenoxide complex $\mathbf{B}$. In this complex, the ortho bromo group is situated close to the palladium, and therefore, oxidative addition to the palladium preferentially occurs at the ortho position. Because the oxidative addition step is considered to be the selectivity-determining step, the ortho-selectivity is realized through this mechanism.

Scheme 4. Mechanism to explain the ortho-selectivity in the site-selective cross-coupling using Ph-HTP.

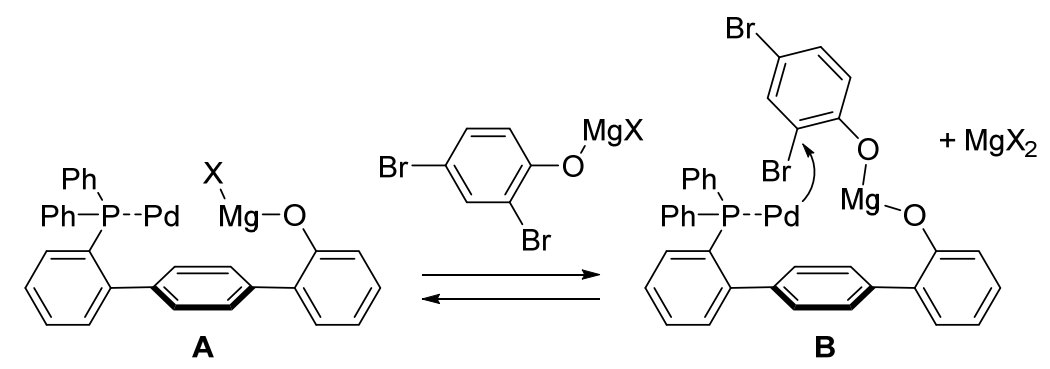

Scheme 5. Site-selective cross-coupling of dibromophenols with Grignard reagents.
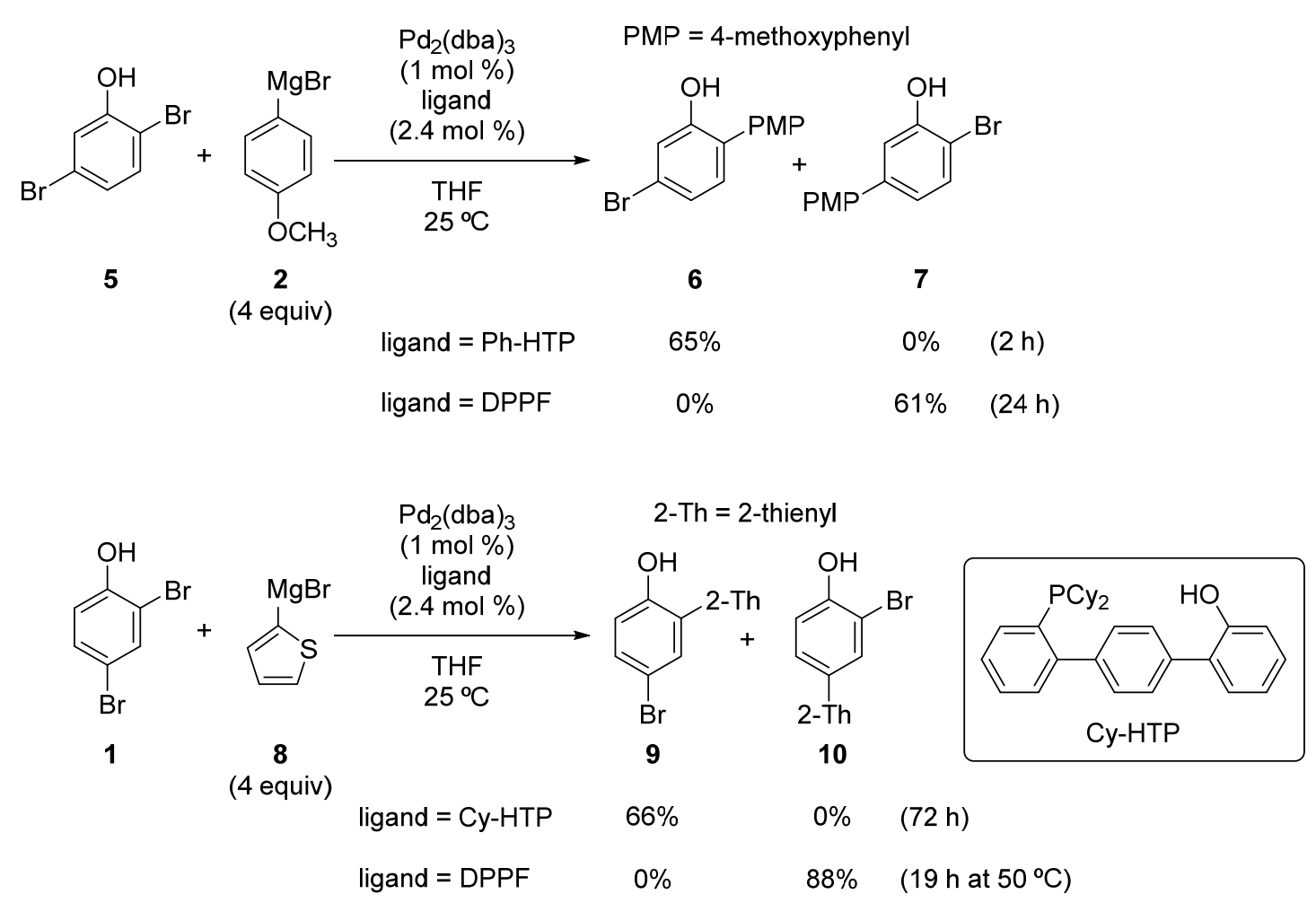
This site-selective cross-coupling was successfully applied to other substrates and Grignard reagents. Representative examples are shown in Scheme 5. Site-selectivity switching was also observed in the reaction of 2,5-dibromophenol (5). It is noteworthy that the Ph-HTP-induced reaction occurred at the sterically more hindered and electronically less reactive (i.e., more electron-rich) position. In the reaction of $\mathbf{1}$ with 2-thienylmagnesium bromide (8), the use of Ph-HTP resulted in a very low yield of 9 for unknown reasons. Fortunately, the use of Cy-HTP, the analogous ligand with cyclohexyl groups on the phosphorus atom, improved the yield greatly.

Although the hydroxyterphenylphosphines described above showed high ortho-selectivity in the reactions of dibromophenols, the substrate scope of the catalytic system was narrow. To improve the ortho-selectivity, we searched for ligands that were more effective and found that the dihydroxyterphenylphosphines, Cy-DHTP and Ph-DHTP, greatly improved the substrate scope [26,27]. For example, when Cy-HTP was used as the ligand, the reaction of 1,6-dibromo-2-naphthol (11) resulted in almost no selectivity (Scheme 6). On the other hand, when Cy-DHTP was used, exclusive selectivity for $\mathbf{1 2}$ over $\mathbf{1 3}$ was observed. The use of Ph-DHTP further improved the result, and 12 was obtained in a $92 \%$ yield. DPPF completely reversed the selectivity, and $\mathbf{1 3}$ was obtained in a high yield. This effectiveness of DHTP over HTP can be attributed to the increased chance for the magnesium phonoxide moiety to be situated close to the palladium.

Scheme 6. Site-selective cross-coupling of 1,6-dibromo-2-naphthol. DHTP, dihydroxyterphenylphosphines.

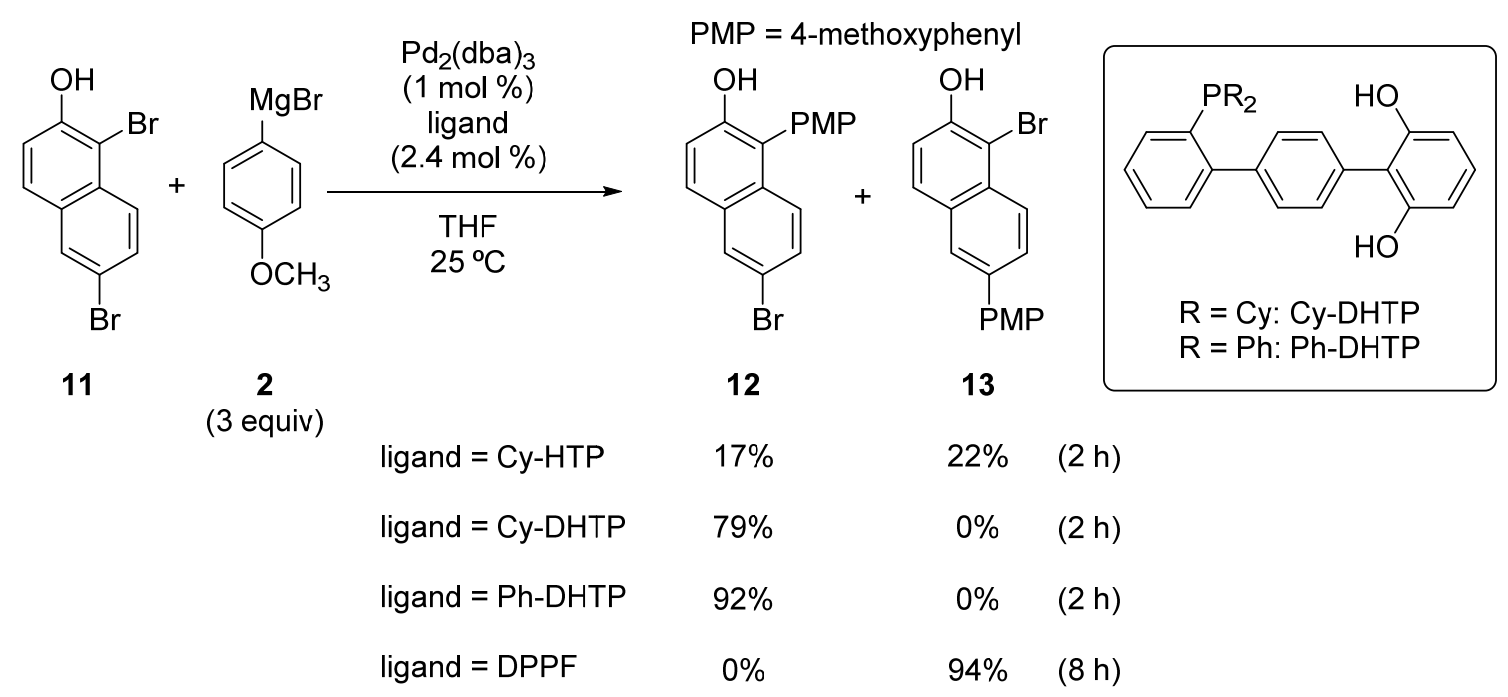

The reaction rate at the position ortho to the hydroxy group is dramatically accelerated by the DHTP ligands [28,29]. Therefore, the reaction can be conducted at lower temperatures to enhance functional group tolerance. As shown in Scheme 7, a Grignard reagent with a $t$-butoxycarbonyl group was prepared from 14 by a reported method at $-40{ }^{\circ} \mathrm{C}$ [30], and the cross-coupling was subsequently conducted at $15^{\circ} \mathrm{C}$. The ester group was tolerated under these conditions, and product $\mathbf{1 5}$ was obtained in a good yield. 
Scheme 7. Site-selective cross-coupling with a Grignard reagent with an ester functionality.

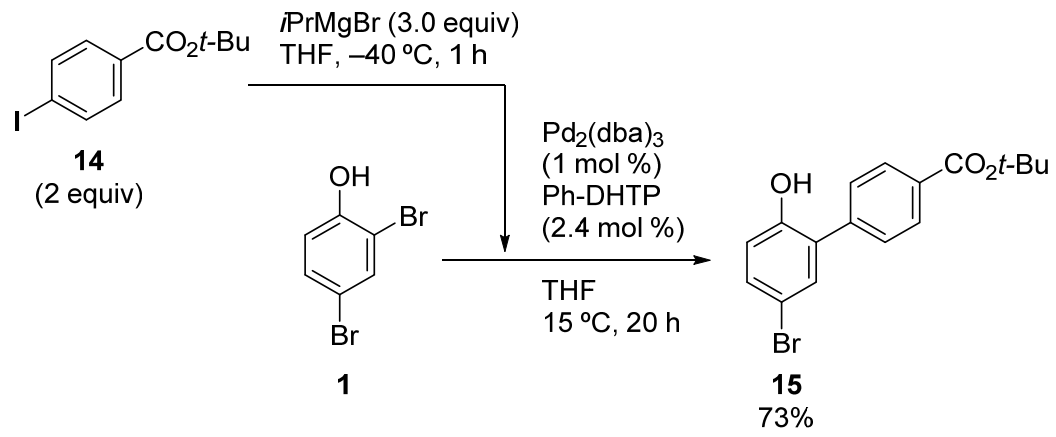

The high ortho-selectivity induced by Ph-DHTP was also demonstrated in the reaction of 4-bromo-2-chlorophenol (16) (Scheme 8). Intriguingly, the effect of Ph-DHTP took priority over the intrinsic reactivity order $(\mathrm{Br}>\mathrm{Cl})$ of the halo groups. Thus, product 3 was obtained in a good yield, whereas 17 was not observed. In the case of a simple phosphine, such as $\mathrm{PCy}_{3}$, the reaction occurred preferentially at the bromo group, as usual.

Scheme 8. Site-selective cross-coupling of 4-bromo-2-chlorophenol.

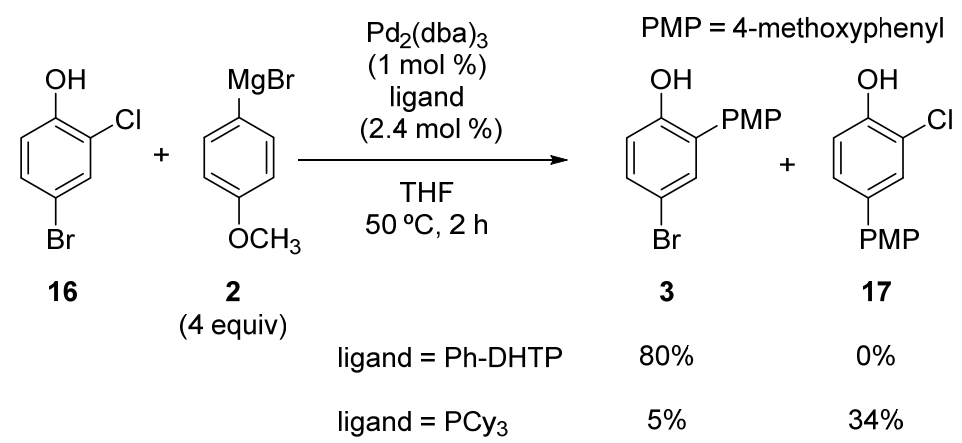

\subsection{Aniline Derivatives}

We applied the catalytic system for the dihalophenols described above to aniline derivatives (Table 1) [26,27]. Dibromoanilines 18-20 and dibromoindole 21 were used as the substrates. As in the case of the phenol derivatives, Ph-DHTP induced excellent selectivity, and the cross-coupling occurred ortho to the NH functionality (Entries 1, 3, 5 and 7, Table 1). We speculated that the mechanism for the selective reaction was similar to the one proposed for dibromophenol (Scheme 4). Unfortunately, satisfactory para-selectivity was not obtained in this system; use of DPPF resulted in poor yields and little selectivity (Entries 2, 4, 6 and 8, Table 1), while the reason for the poor results is unclear.

\subsection{Benzoic Acid Derivatives}

Houpis and coworkers reported the site-selective Suzuki-Miyaura coupling [31] of 2,4-dibromobenzoic acid (22) with arylboronic acids (Scheme 9) [32]. When $\mathrm{Pd}_{2}(\mathrm{dba})_{3}$ was used under phosphine-free conditions, ortho-selective cross-coupling occurred, and product 24 was obtained with excellent selectivity. This selectivity was presumably a result of the coordination of the carboxylate anion to Pd, as such coordination situates the ortho bromo group close to the Pd. The choice of an appropriate base 
and solvent ( $\mathrm{LiOH}$ and $N$-methylpyrrolidone $(\mathrm{NMP}) / \mathrm{H}_{2} \mathrm{O}$ ) was crucial for the high yield and selectivity. On the other hand, the use of bulky bidentate phosphines, such as bis[2-(diphenylphosphino)phenyl] ether (DPEPhos), reversed the selectivity, and isomer 25 was preferentially formed. The phosphine is assumed to disrupt the coordination of the carboxylate to Pd, thereby leading to the observed para-selectivity.

Table 1. Site-selective cross-coupling of dibromoaniline derivatives and dibromoindole.

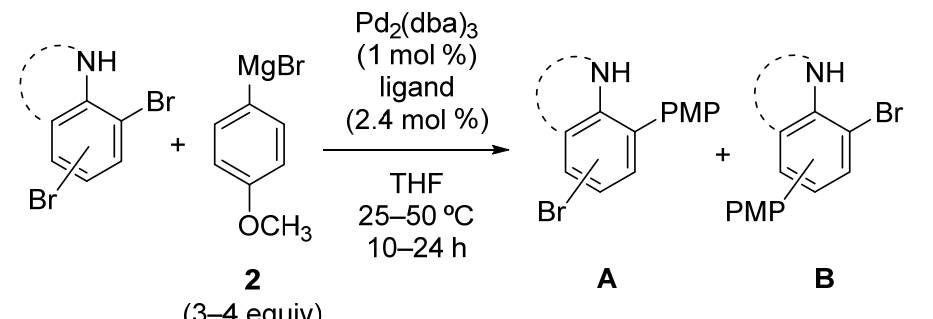

\begin{tabular}{|c|c|c|c|c|}
\hline \multirow{2}{*}{ Entry } & \multirow{2}{*}{ Substrate } & \multirow{2}{*}{ Ligand } & \multicolumn{2}{|c|}{ Yield $(\%)$} \\
\hline & & & $\mathbf{A}$ & B \\
\hline 1 & & Ph-DHTP & 90 & 0 \\
\hline 2 & 18 & DPPF & 9 & 15 \\
\hline 3 & & Ph-HTP & 63 & 0 \\
\hline 4 & 19 & DPPF & 15 & 32 \\
\hline 5 & & Ph-DHTP & 70 & 0 \\
\hline 6 & 20 & DPPF & 29 & 21 \\
\hline 7 & & Ph-DHTP & 81 & 0 \\
\hline 8 & 21 & DPPF & 0 & 37 \\
\hline
\end{tabular}

Scheme 9. Site-selective Suzuki-Miyaura coupling of 2,4-dibromobenzoic acid.

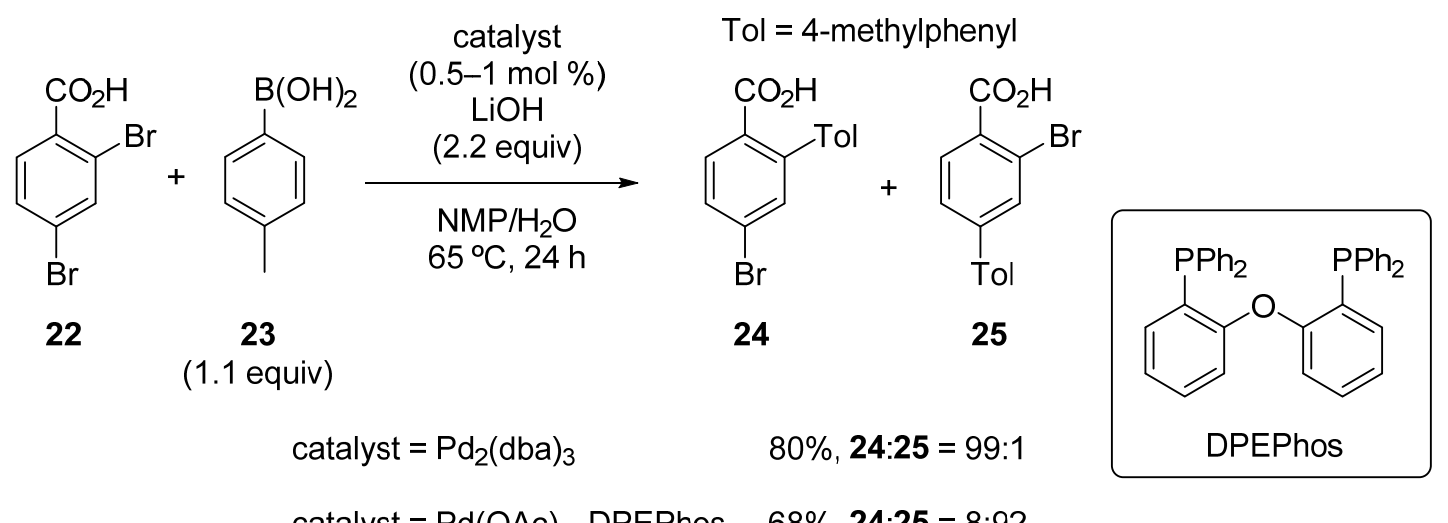

catalyst $=\mathrm{Pd}(\mathrm{OAc})_{2}-\mathrm{DPEPhos} \quad 68 \%, \mathbf{2 4 : 2 5}=\mathbf{8 : 9 2}$ 


\section{Dihaloheteroarenes}

\subsection{Pyrone Derivatives}

In 2003, Cho and coworkers reported the site-selective Migita-Kosugi-Stille coupling [33,34] of 3,5-dibromo-2-pyrone (26) with tributylphenyltin (27) (Table 2) [35]. When the reaction was conducted in toluene, the cross-coupling preferentially occurred at the $\mathrm{C} 3$ position to give $\mathbf{2 8}$, regardless of the amount of CuI used (Entries 1-3, Table 2). In DMF, however, the site-preference was affected by the number of equivalents of $\mathrm{CuI}$; in the presence of 1.0 equiv of $\mathrm{CuI}, 29$ was obtained with excellent selectivity (Entry 7, Table 2). Even when 0.5 equiv of $\mathrm{CuI}$ was used, the switch of the selectivity was observed (Entry 6, Table 2). Therefore, while the same catalyst $\left(\operatorname{Pd}\left(\mathrm{PPh}_{3}\right)_{4}\right)$ was used, a different catalytic species may have been formed in the presence of $\mathrm{CuI}$. To gain insight into the mechanism of this selectivity, the oxidative addition step was separately investigated by conducting the reaction without the tin compound. It was revealed that in toluene with or without CuI or in DMF without CuI, oxidative addition occurred exclusively at the $\mathrm{C} 3$ position. Conversely, in DMF with one equiv of $\mathrm{CuI}$, oxidative addition at the C5 position was preferred. In addition, the palladium complex formed through the $\mathrm{C} 5$ oxidative addition was found to undergo a much faster reaction with tributylphenyltin than the complex formed through the $\mathrm{C} 3$ oxidative addition. Thus, not only the preferred position for oxidative addition, but also the subsequent faster reaction accounts for the C5-selective coupling.

Table 2. Site-selective Migita-Kosugi-Stille coupling of 3,5-dibromo-2-pyrone.

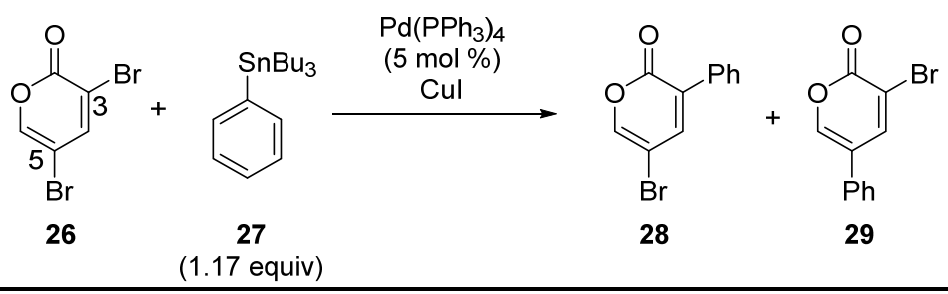

\begin{tabular}{cclcc}
\hline \multirow{2}{*}{ Entry } & \multirow{2}{*}{ CuI (equiv) } & Conditions & \multicolumn{2}{c}{ Yield (\%) } \\
\cline { 4 - 5 } & 0 & toluene, $100^{\circ} \mathrm{C}, 0.5 \mathrm{~h}$ & 81 & trace \\
\hline 1 & 0.1 & toluene, $100^{\circ} \mathrm{C}, 0.5 \mathrm{~h}$ & 94 & trace \\
2 & 1.0 & toluene, $100{ }^{\circ} \mathrm{C}, 2 \mathrm{~h}$ & 71 & 6 \\
3 & 0 & DMF, $50{ }^{\circ} \mathrm{C}, 4$ days & 41 & 2 \\
4 & 0.1 & $\mathrm{DMF}, 50{ }^{\circ} \mathrm{C}, 5 \mathrm{~h}$ & 34 & 20 \\
5 & 0.5 & $\mathrm{DMF}, 50^{\circ} \mathrm{C}, 2.5 \mathrm{~h}$ & trace & 64 \\
6 & 1.0 & $\mathrm{DMF}, 50^{\circ} \mathrm{C}, 2 \mathrm{~h}$ & trace & 75 \\
7 & & &
\end{tabular}

\subsection{Pyridine Derivatives}

In 2003, Yang and coworkers reported an example of site-selectivity switching in the Suzuki-Miyaura coupling of 2,6-dichloropyridine derivatives 30 with phenylboronic acid (31) (Table 3) [36]. When $\mathrm{Pd}\left(\mathrm{PPh}_{3}\right)_{4}$ was used as the catalyst, $30(R=\mathrm{OMe})$ was preferentially converted to $\mathbf{3 3}$, in which the phenyl group was introduced at the less hindered C6 position (Entry 1, Table 3). However, when PXPd2 [37] was used, compound 32 was the major product (Entry 2, Table 3), and methanol was 
found to be the best solvent. This preference for the $\mathrm{C} 2$ position, which is attributable to the coordination of the carbonyl oxygen to the catalytically active $\mathrm{Pd}(0)$ species, was further enhanced by replacing the ester of $\mathbf{3 0}$ with an amide group (Entry 3, Table 3). While the selectivity is not high and largely depends on the neighboring group, this early example demonstrated the possibility of site-selective cross-coupling of nitrogen-containing heterocycles.

Table 3. Site-selective Suzuki-Miyaura coupling of 2,6-dichloropyridine derivatives.<smiles>[R]C(=O)c1ccc(Cl)nc1Cl</smiles><smiles>Oc1ccccc1</smiles>

30 31 (1-1.2 equiv)<smiles>[R]C(=O)c1ccc(Cl)nc1[PH3+]</smiles>

32<smiles>[R]C(=O)c1ccc(-c2ccccc2)nc1Cl</smiles>

33

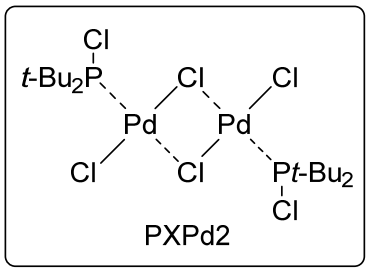

PXPd2

\begin{tabular}{ccccc}
\hline Entry & $\mathbf{R}$ & Catalyst (mol \%) & Conditions & $\mathbf{3 2 : 3 3}$ \\
\hline 1 & $\mathrm{OMe}$ & $\mathrm{Pd}\left(\mathrm{PPh}_{3}\right)_{4}(5)$ & $\mathrm{THF}$, reflux, 16 h & $1: 5 *$ \\
2 & $\mathrm{OMe}$ & $\mathrm{PXPd} 2(1)$ & $\mathrm{MeOH}$, reflux, 30 min & $2.5: 1 *$ \\
3 & $\mathrm{NHCH}_{2} \mathrm{CH}_{2} \mathrm{OPh}$ & $\mathrm{PXPd} 2(1)$ & $\mathrm{MeOH}, 55^{\circ} \mathrm{C}, 1 \mathrm{~h}$ & $9: 1 * *$ \\
\hline
\end{tabular}

* Yields are not indicated; ** the yield of 32 is $61 \%$.

Dai, Chen and coworkers developed the ligand-dependent site-selective Suzuki-Miyaura coupling of 2,4-dichloropyridine (34) (Table 4) [38]. For this substrate, the reaction at the C2 position was expected to be preferred according to the calculations of bond dissociation energies [19]. In fact, a DPPF-based catalyst exclusively gave 2-phenylated product 36 (Entry 1, Table 4). Interestingly, the use of 1,2,3,4,5-pentaphenyl-1'-(di-t-butylphosphino)ferrocene (Q-Phos) [39] as the ligand switched the site-selectivity (Entry 2, Table 4). Under the optimized conditions (KF as the base and toluene as the solvent), a 2.4:1 ratio of $\mathbf{3 5 : 3 6}$ was obtained. It is noteworthy that site-selectivity was achieved for these coupling reactions with $\mathbf{3 4}$, which is a substrate without a directing substituent.

Table 4. Site-selective Suzuki-Miyaura coupling of 2,4-dichloropyridine. Q-Phos, 1,2,3,4,5-pentaphenyl-1'-(di-t-butylphosphino)ferrocene

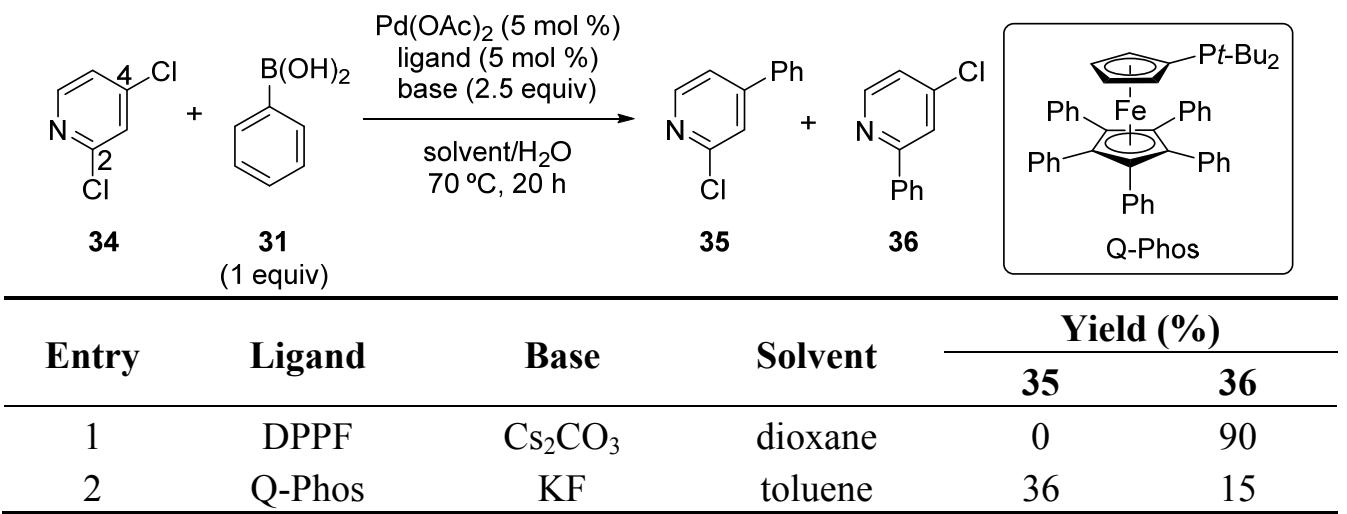

\subsection{Pyridazine Derivatives}

Dai, Chen and coworkers also reported the site-selectivity switching in the Suzuki-Miyaura coupling of dichloropyridazines [38]. The reaction of 3,5-dichloropyridazine (37) with phenylboronic 
acid in the presence of a DPPF-based catalyst predominantly occurred at the C3 position to give 39 (Scheme 10), coincident with the calculated bond dissociation energies. Selectivity switching was observed when Q-Phos was used instead of DPPF, and $\mathbf{3 8}$ was obtained with high site-selectivity. Various other substrates were successfully used for the site-selective reactions (Scheme 11), and the major products were isolated in a $53 \%-92 \%$ yield.

Scheme 10. Site-selective Suzuki-Miyaura coupling of 3,5-dichloropyridazine. Yields are not indicated.

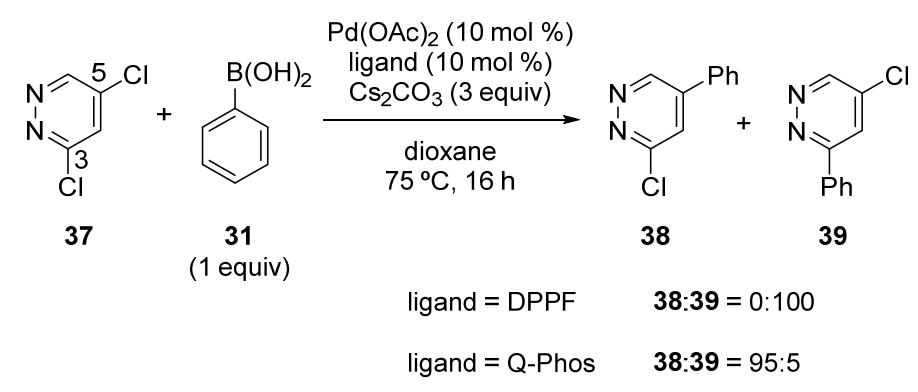

Scheme 11. Examples of site-selective Suzuki-Miyaura coupling of 3,5-dichloropyridazines.
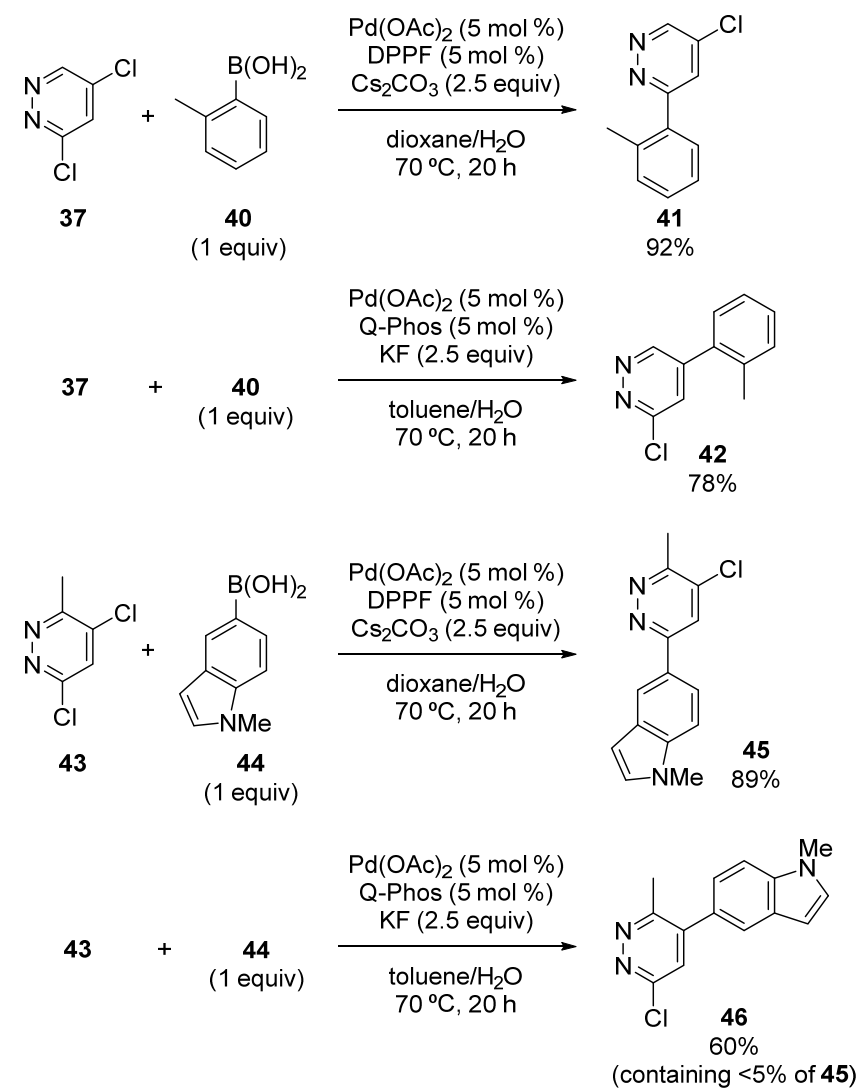

\subsection{Oxazole Derivatives}

Strotman, Chobanian and coworkers developed the catalyst-controlled, site-selective Suzuki-Miyaura coupling of 2,4-diiodooxazole (47) with phenylboronic acid (Scheme 12) [40]. The use of highly electron-rich phosphines as ligands tended to afford C2-phenylated product 49. 1,3,5-Triaza-7-phosphaadamantane (50) was found to be exceptionally effective and afforded 49 with 
high site-selectivity. Xantphos, on the other hand, preferentially gave C4-phenylated product 48. Fluorophenylboronic acid (51) also reacted with high selectivity. A variety of boronic acids were successfully used for these catalytic systems.

Scheme 12. Site-selective Suzuki-Miyaura coupling of 2,4-diiodooxazole.

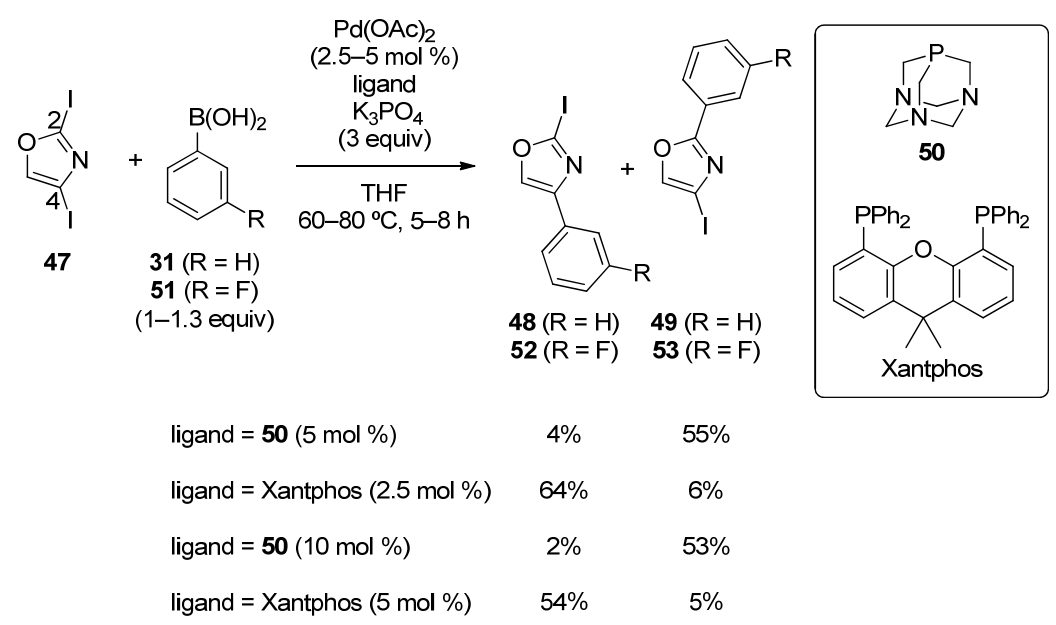

\subsection{Imidazole Derivatives}

Strotman, Chobanian and coworkers also reported site-selectivity switching in the Suzuki-Miyaura coupling of 2,5-dihalo-1-methylimidazoles [40]. A representative example is shown in Scheme 13. As in the case of the oxazole derivative, phosphine $\mathbf{5 0}$ preferentially afforded C2-arylated product $\mathbf{5 7}$. For C5-selective coupling, phosphine $\mathbf{5 8}$ was found to be effective. The same authors also revealed that 2,4- and 2,5-dibromothiazoles selectively reacted at the $\mathrm{C} 2$ position under xantphos-based catalysis.

Scheme 13. Site-selective Suzuki-Miyaura coupling of 2,5-dibromo-1-methylimidazole.

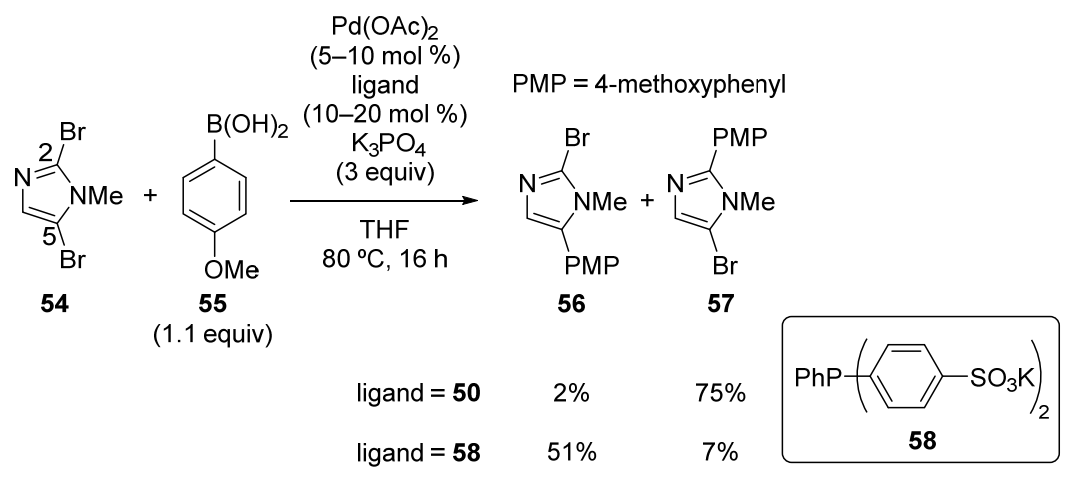

\section{Conclusions}

Palladium-catalyzed site-selective cross-coupling of dihaloarenes provides an efficient approach to the introduction of substituents at specific positions of benzene and heterocycle derivatives. The halo group remaining after the mono-cross-coupling can further be converted to other substituents. Although the examples are still limited at this stage and often depend on neighboring group effects, the easy availability of the starting materials and the versatility of the methodology will make it practical and useful for the rapid production of diverse arenes with multiple substituents. While the origin of the 
site-selectivity in many cases is presently unclear, further studies will uncover the mechanisms underlying the selective cross-coupling. We are happy to see the progress that has been made, as described in this review, and are anticipating more in the future of this exciting field.

\section{Author Contributions}

The literature was researched by both of the authors. Kei Manabe wrote the first draft of the manuscript that was then improved by Miyuki Yamaguchi.

\section{Conflicts of Interest}

The authors declare no conflict of interest.

\section{References}

1. Negishi, E.-i. Handbook of Organopalladium. Chemistry for Organic Synthesis; John Wiley \& Sons Ltd.: New York, NY, USA, 2002.

2. De Meijere, A.; Diederich, F. Metal-Catalyzed Cross-Coupling Reactions, 2nd ed.; Wiley-VCH: Weinheim, Germany, 2004.

3. Tsuji, J. Palladium Reagents and Catalysts; John Wiley \& Sons: West Sussex, UK, 2004.

4. Unrau, C.M.; Campbell, M.G.; Snieckus, V. Directed ortho metalation-suzuki cross coupling connections. convenient regiospecific routes to functionalized $m$ - and $p$-Teraryls and $m$-Quinquearyls. Tetrahedron Lett. 1992, 33, 2773-2776.

5. Goodby, J.W.; Hird, M.; Lewis, R.A.; Toyne, K.J. 5-Bromo-2-iodopyrimidine: A novel, useful intermediate in selective palladium-catalysed cross-coupling reactions for efficient convergent syntheses. Chem. Commun. 1996, 2719-2720.

6. Heinrich, A.C.J.; Thiedemann, B.; Gates, P.J.; Staubitz, A. Dual selectivity: Electrophile and nucleophile selective cross-coupling reactions on a single aromatic substrate. Org. Lett. 2013, 15, 4666-4669.

7. Montoir, D.; Tonnerre, A.; Duflos, M.; Bazin, M.-A. Differential functionalization of 1,6-Naphthyridin-2(1H)-ones through sequential one-pot suzuki-miyaura cross-couplings. Eur. J. Org. Chem. 2014, 1487-1495.

8. Kamikawa, T.; Hayashi, T. Control of reactive site in palladium-catalyzed grignard cross-coupling of arenes containing both bromide and triflate. Tetrahedron Lett. 1997, 38, 7087-7090.

9. Littke, A.F.; Dai, C.; Fu, G.C. Versatile catalysts for the suzuki cross-coupling of arylboronic acids with aryl and vinyl halides and triflates under mild conditions. J. Am. Chem. Soc. 2000, 122, 4020-4028.

10. Ashcroft, C.P.; Fussell, S.J.; Wilford, K. Catalyst controlled regioselective suzuki cross-coupling of 2-(4-bromophenyl)-5-chloropyrazine. Tetrahedron Lett. 2013, 54, 4529-4532.

11. Yamamoto, Y.; Seko, T.; Nemoto, H. New mmethod for the synthesis of boron-10 containing nucleoside derivatives for neutron-capture therapy via palladium-catalyzed reaction. J. Org. Chem. 1989, 54, 4734-4736. 
12. Schröter, S.; Stock, C.; Bach, T. Regioselective cross-coupling reactions of multiple halogenated nitrogen-, oxygen-, and sulfur-containing heterocycles. Tetrahedron 2005, 61, 2245-2267.

13. Fairlamb, I.J.S. Regioselective (site-selective) functionalization of unsaturated halogenated nitrogen, oxygen and sulfur heterocycles by pd-catalysed cross-couplings and direct arylation processes. Chem. Soc. Rev. 2007, 36, 1036-1045.

14. Wang, J.-R.; Manabe, K. Transition-Metal-Catalyzed site-selective cross-coupling of di- and polyhalogenated compounds. Synthesis 2009, 1405-1427.

15. Hassan, Z.; Patonay, T.; Langer, P. Regioselective suzuki-miyaura reactions of aromatic bis-triflates: Electronic versus steric effects. Synlett 2013, 24, 412-423.

16. Singh, R.; Just, G. Rates and regioselectivities of the palladium-catalyzed ethynylation of substituted bromo- and dibromobenzenes. J. Org. Chem. 1989, 54, 4453-4457.

17. Handy, S.T.; Zhang, Y. A simple guide for predicting regioselectivity in the coupling of polyhaloheteroaromatics. Chem. Commun. 2006, 299-301.

18. Legault, C.Y.; Garcia, Y.; Merlic, C.A.; Houk, K.N. Origin of regioselectivity in palladium-catalyzed cross-coupling reactions of polyhalogenated heterocycles. J. Am. Chem. Soc. 2007, 129, 12664-12665.

19. Garcia, Y.; Schoenebeck, F.; Legault, C.Y.; Merlic, C.A.; Houk, K.N. Theoretical bond dissociation energies of halo-heterocycles: Trends and relationships to regioselectivity in palladium-catalyzed cross-coupling reactions. J. Am. Chem. Soc. 2009, 131, 6632-6639.

20. Newman, S.G.; Lautens, M. The role of reversible oxidative addition in selective palladium(0)catalyzed intramolecular cross-couplings of polyhalogenated substrates: Synthesis of brominated indoles. J. Am. Chem. Soc. 2010, 132, 11416-11417.

21. Ishikawa, S.; Manabe, K. Ortho-Selective cross coupling of dibromophenols and dibromoanilines with grignard reagents in the presence of palladium catalysts bearing hydroxylated oligoarene-type phosphine. Chem. Lett. 2007, 36, 1304-1305.

22. Tamao, K.; Sumitani, K.; Kumada, M. Selective carbon-carbon bond formation by cross-coupling of grignard reagents with organic halides. Catalysis by nickel-phosphine complexes. J. Am. Chem. Soc. 1972, 94, 4374-4376.

23. Corriu, R.J.P.; Masse, J.P. Activation of grignard reagents by transition-metal complexes. A new and simple synthesis of trans-stilbenes and polyphenyls. J. Chem. Soc. Chem. Commun. 1972, 144a.

24. Ishikawa, S.; Manabe, K. Oligoarene strategy for catalyst development. hydroxylated oligoarene-type phosphines for palladium-catalyzed cross coupling. Chem. Lett. 2007, 36, 1302-1303.

25. Ishikawa, S.; Manabe, K. Synthesis of hydroxylated oligoarene-type phosphines by a repetitive two-step method. Tetrahedron 2010, 66, 297-303.

26. Ishikawa, S.; Manabe, K. DHTP ligands for the highly ortho-selective, palladium-catalyzed cross-coupling of dihaloarenes with grignard reagents: A conformational approach for catalyst improvement. Angew. Chem. Int. Ed. 2010, 49, 772-775.

27. Ishikawa, S.; Manabe, K. Hydroxylated terphenylphosphine ligands for palladium-catalyzed ortho-selective cross-coupling of dibromophenols, dibromoanilines, and their congeners with grignard reagents. Tetrahedron 2011, 67, 10156-10163. 
28. Yamaguchi, M.; Katsumata, H.; Manabe, K. One-Pot synthesis of substituted benzo[b]furans from mono- and dichlorophenols using palladium catalysts bearing dihydroxyterphenylphosphine. $J$. Org. Chem. 2013, 78, 9270-9281.

29. Yamaguchi, M.; Manabe, K. One-Pot synthesis of 2,4-disubstituted indoles from $n$-tosyl-2,3dichloroaniline using palladium-dihydroxyterphenylphosphine catalyst. Org. Lett. 2014, 16, 2386-2389.

30. Boymond, L.; Rottländer, M.; Cahiez, G.; Knochel, P. Preparation of highly functionalized grignard reagents by an iodine-magnesium exchange reaction and its application in solid-phase synthesis. Angew. Chem. Int. Ed. 1998, 37, 1701-1703.

31. Suzuki, A. Cross-Coupling reactions of organoboranes: An easy way to construct $\mathrm{C}-\mathrm{C}$ bonds (Nobel Lecture). Angew. Chem. Int. Ed. 2011, 50, 6722-6737.

32. Houpis, I.N.; Huang, C.; Nettekoven, U.; Chen, J.G.; Liu, R.; Canters, M. Carboxylate directed cross-coupling reactions in the synthesis of trisubstituted benzoic acids. Org. Lett. 2008, 10, 5601-5604.

33. Kosugi, M.; Sasazawa, K.; Shimizu, Y.; Migita, T. Reactions of allyltin compounds III. Allylation of aromatic halides with allyltributyltin in the presence of Tetrakis(triphenylphosphine)palladium( 0$)$. Chem. Lett. 1977, 6, 301-302.

34. Milstein, D.; Stille, J.K. A general, selective, and facile method for ketone synthesis from acid chlorides and organotin compounds catalyzed by palladium. J. Am. Chem. Soc. 1978, 100, 3636-3638.

35. Kim, W.-S.; Kim, H.-J.; Cho, C.-G. Regioselectivity in the stille coupling reactions of 3,5-dibromo-2-pyrone. J. Am. Chem. Soc. 2003, 125, 14288-14289.

36. Yang, W.; Wang, Y.; Corte, J.R. Efficient synthesis of 2-Aryl-6-chloronicotinamides via PXPd2-Catalyzed regioselective suzuki coupling. Org. Lett. 2003, 5, 3131-3134.

37. Li, G.Y. Catalysis using phosphine oxide and phosphine sulfide complexes with Pd and Ni for the synthesis of biaryls and arylamines. WO2002000574A2, 3 January 2002.

38. Dai, X.; Chen, Y.; Garrell, S.; Liu, H.; Zhang, L.-K.; Palani, A.; Hughes, G.; Nargund, R. Ligand-Dependent site-selective suzuki cross-coupling of 3,5-dichloropyridazines. J. Org. Chem. 2013, 78, 7758-7763.

39. Shelby, Q.; Kataoka, N.; Mann, G.; Hartwig, J. Unusual in situ ligand modification to generate a catalyst for room temperature aromatic $\mathrm{C}-\mathrm{O}$ bond formation. J. Am. Chem. Soc. 2000, 122, 10718-10719.

40. Strotman, N.A.; Chobanian, H.R.; He, J.; Guo, Y.; Dormer, P.G.; Jones, C.M.; Steves, J.E. Catalyst-controlled regioselective suzuki couplings at both positions of dihaloimidazoles, dihalooxazoles, and dihalothiazoles. J. Org. Chem. 2010, 75, 1733-1739.

(C) 2014 by the authors; licensee MDPI, Basel, Switzerland. This article is an open access article distributed under the terms and conditions of the Creative Commons Attribution license (http://creativecommons.org/licenses/by/3.0/). 